\title{
Maternal obesity affects neonatal adipogenesis
}

Umbilical-cord-derived mesenchymal stem cells (MSCs) from neonates born to mothers with obesity have increased adipogenic potential, according to a new study published in Diabetes.

Maternal obesity during pregnancy is associated with increased birth weight and neonatal fat mass. Epidemiological studies have shown that children born to women with obesity are at increased risk of obesity in later life, independent of their postnatal lifestyles. On the basis of studies in sheep suggesting that maternal obesity alters fetal skeletal muscle and adipose tissue development, the investigators hypothesized that exposure to maternal obesity would alter the lineage specification of fetal MSCs, which differentiate into myocytes and adipocytes. "Fetal umbilical cord tissue is an abundant source of MSCs," explains corresponding author Kristen Boyle. "We obtained fetal MSCs from umbilical cord tissue samples from babies born to women who were obese or normal weight, with the hypothesis that phenotypic differences would be evident."

At age $24-48 \mathrm{~h}$, babies born to women with obesity $(n=14)$ had a greater proportion of fat mass than babies born to normal-weight mothers $(n=15)$. Umbilical cord MSCs from babies exposed to obesity in utero showed increased lipid accumulation and expression of adipogenesis markers, yet no change in levels of myogenesis markers. The researchers found that levels of $\beta$-catenin protein, which inhibits adipogenesis and promotes myogenesis, were reduced in MSCs from neonates born to women with obesity. Nuclear $\beta$-catenin content was normalized in these cells by inhibiting GSK3 $\beta$, resulting in reduced nuclear content of proadipogenic PPAR $\gamma$. "We have planned long-term follow-up of the children from which these cells were derived to better understand whether these MSC characteristics are informative of future adiposity," concludes Boyle.

Hugh Thomas

ORIGINAL ARTICLE Boyle, K. E. et al.

Mesenchymal stem cells from infants born to obese mothers exhibit greater potential for adipogenesis: the Healthy Start BabyBUMP project. Diabetes http://dx.doi.org/10.2337/db15-0849 